Journal for ImmunoTherapy of Cancer

\section{Integrated immunological analysis of a successful conversion of locally advanced hepatocellular carcinoma to resectability with neoadjuvant therapy}

To cite: Ho WJ, Sharma G, Zhu Q, et al. Integrated immunological analysis of a successful conversion of locally advanced hepatocellular carcinoma to resectability with neoadjuvant therapy. Journal for ImmunoTherapy of Cancer 2020;8:e000932. doi:10.1136/ jitc-2020-000932

- Additional material is published online only. To view please visit the journal online (http://dx.doi.org/10.1136/jitc2020-000932).

Accepted 26 August 2020
Check for updates

(C) Author(s) (or their employer(s)) 2020. Re-use permitted under CC BY-NC. No commercial re-use. See rights and permissions. Published by BMJ.

For numbered affiliations see end of article.

Correspondence to

Mark Yarchoan;

mark.yarchoan@jhmi.edu

\section{ABSTRACT}

Hepatocellular carcinoma (HCC) is the fourth leading cause of cancer death worldwide with a minority of patients being diagnosed early enough for curative-intent interventions. We report the first use of preoperative cabozantinib plus nivolumab to successfully downstage what presented as unresectable HCC as part of an ongoing phase $1 \mathrm{~b}$ study. Preoperative treatment with cabozantinib and nivolumab led to $>99 \%$ reduction in alpha-fetoprotein, $-37.3 \%$ radiographic reduction by RECIST 1.1 and a near complete pathologic response ( $80 \%$ to $100 \%$ necrosis). An integrated immunological analysis was performed on the post-treatment surgical tumor sample and matched pre-treatment and posttreatment peripheral blood samples with high-dimensional imaging and cytometry techniques. Bayesian non-negative matrix factorization (CoGAPS, Coordinated Gene Activity in Pattern Sets) and self-organizing map (FlowSOM) algorithms were used to distinguish changes in functional markers across cellular neighborhoods in the single cell data sets. Brisk immunological infiltration into the tumor microenvironment was observed in non-random, organized cellular neighborhoods. Systemically, combination therapy led to marked promotion of effector cytotoxic T cells and effector memory helper T cells. Natural killer cells also increased with therapy. The patient remains without disease recurrence and with a normal alpha-fetoprotein approximately 2 years from presentation. Our study provides proof-of-concept that borderline resectable or locally advanced HCC warrants consideration of downstaging with effective neoadjuvant systemic therapy for subsequent curative resection.

\section{INTRODUCTION}

Hepatocellular carcinoma (HCC) is the fourth leading cause of cancer death worldwide. The estimated 5-year survival rate for $\mathrm{HCC}$ is $18 \%$, reflecting that a minority of patients are diagnosed early enough to be candidates for curative-intent therapies such as surgical resection, ablation, or liver transplantation. ${ }^{1}$ Patients who are not transplant candidates and who have tumors outside of resection criteria at presentation are generally treated with locoregional therapy or systemic therapy with palliative intent. The utility of preoperative transcatheter arterial chemoembolization for HCG remains controversial at best with uncontrolled series as well as randomized controlled trial studies having demonstrated associations with an increased risk of postoperative disease recurrence. ${ }^{2-6}$ Moreover, the use of systemic therapy to downstage HCC has not been feasible due to the low response rate of sorafenib, which was until recently the only approved systemic therapy. Novel combinations of targeted agents and inhibitors of the programmed cell death protein 1 (PD-1) axis demonstrate response rates of approximately $30 \%$ in $\mathrm{HCC}^{7-9}$ which is similar to tumor types for which neoadjuvant systemic therapy is standard practice in borderline resectable or locally advanced cases. ${ }^{10}{ }^{11} \mathrm{We}$ report the first patient treated on a protocol of neoadjuvant cabozantinib plus nivolumab in borderline resectable or locally advanced HCC (NCT03299946) who was successfully converted to a resection candidate, with prolonged disease-free survival after resection. Cabozantinib is a tyrosine kinase inhibitor (TKI) with potent activity against VEGFR2, MET, AXL, and other kinases. Nivolumab is an inhibitor of the PD-1 immune checkpoint.

Recent progress in single-cell technologies, for example, in suspension cytometry, sequencing, imaging methods, and analytical algorithms, have enabled unprecendented depth and breadth to studying immunologic phenomena. For example, imaging mass cytometry and spatial analysis tools have recently emerged as powerful tools for evaluating spatial coordination of cells within the tumor microenvironment. ${ }^{12}$ We have recently developed and demonstrated the 
utility of a non-negative matrix factorization algorithm, Coordinated Gene Activity in Pattern Sets (CoGAPS), to discover meaningful cell types and states from single-cell data sets. ${ }^{13}$ Whereas a clustering algorithm based on SelfOrganizing Map, for example, FlowSOM ${ }^{14}$ limits cells to a single cluster, protein markers from CoGAPS can associate cells with both functional markers and discrete cell types. To profile the immunological state underlying the clinical outcome in response to the novel combination of cabozantinib and nivolumab, we employ these highly multiplexed imaging and cytometry assays along with the computational tools to provide an in-depth, unbiased analysis of the immunological response.

\section{RESULTS}

\section{Clinical response}

A man in his early 70s with a history of hepatitis C cirrhosis (Child Pugh A) presented with a $5.8 \mathrm{~cm}$ hypervascular and infiltrative lesion in his inferior right hepatic lobe with extension into the porta hepatis, and tumor thrombus extending into the right anterior portal vein, consistent with HCC. Also in the right lobe was a second focus of enhancement that was poorly defined but most consistent with a satellite lesion. The disease was deemed unresectable based on Barcelona Clinic Liver Cancer criteria. ${ }^{15}$ His alpha-fetoprotein (AFP) increased from
$17,528 \mathrm{ng} / \mathrm{mL}$ at the time of presentation to $30,070 \mathrm{ng} /$ $\mathrm{mL}$ at the time of multidisciplinary evaluation 12 days later (AFP doubling time of approximately 15 days). He was treated with cabozantinib $40 \mathrm{mg}$ oral daily for 2 weeks (cabozantinib lead in), followed by 8 weeks of concurrent cabozantinib $40 \mathrm{mg}$ plus nivolumab (240 mg every 2 weeks for a total of four doses). Additional details related to the clinical trial are available in the online supplemental information. The subject's AFP declined with therapy to $12.9 \mathrm{ng} / \mathrm{mL}$ ( $>99 \%$ reduction from treatment baseline) and the patient experienced a $37.3 \%$ radiographic reduction in his lesions by RECIST 1.1 (figure 1A,B).

Approximately 4 weeks after his last dose of nivolumab, he underwent a right hepatectomy and cholecystectomy. The operation itself was uneventful, although the high bifurcation of the portal vein and the patient's original tumor thrombus and macrovascular invasion made it difficult to get around the right portal vein during the surgical operation. His postoperative course was complicated by significant output from his Jackson-Pratt drain, and the patient received intermittent doses of intravenous furosemide to manage the drain output. In the opinion of the treating surgical oncologist $(\mathrm{MJW})$, there were no discernable adverse events from neoadjuvant therapy on the perioperative course, and the patient's wound healing was normal. Pathology from the surgical resection
A
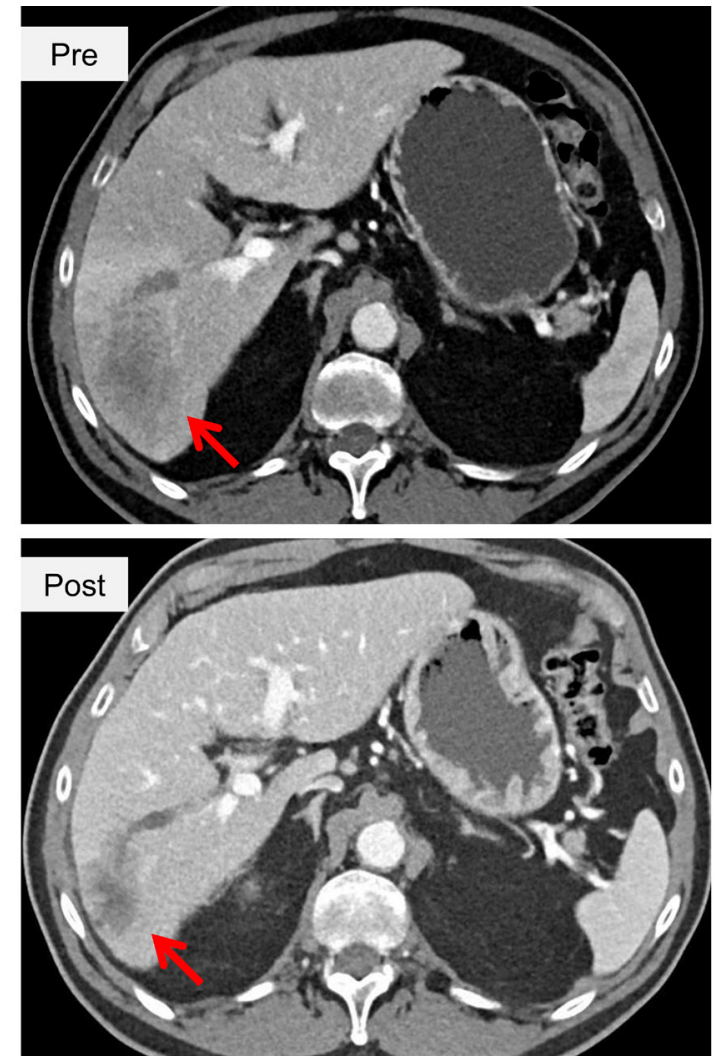

B

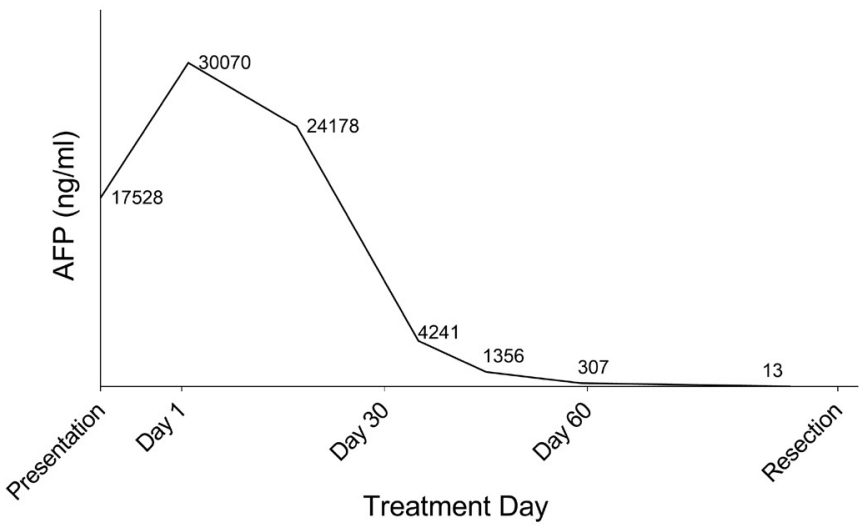

C

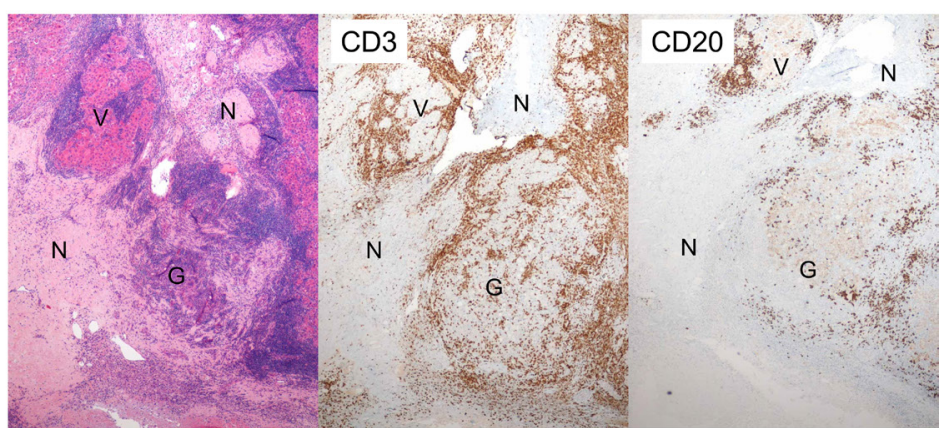

$\mathrm{V}=$ viable tumor, $\mathrm{N}=$ necrosis, $\mathrm{G}=$ granulomatous inflammation

Figure 1 Clinical response to neoadjuvant cabozantinib and nivolumab. Neoadjuvant therapy resulted in (A) tumor downstaging, with the dominant infiltrative lesion decreasing from $5.81 \mathrm{~cm}$ to $3.64 \mathrm{~cm}$ in maximum diameter (B) marked reduction in AFP, and $(C)$ tumor necrosis and brisk immune infiltration by immunohistochemistry with $C D 3+T$ cells and $C D 20+B$ cells. AFP, alpha-fetoprotein. 

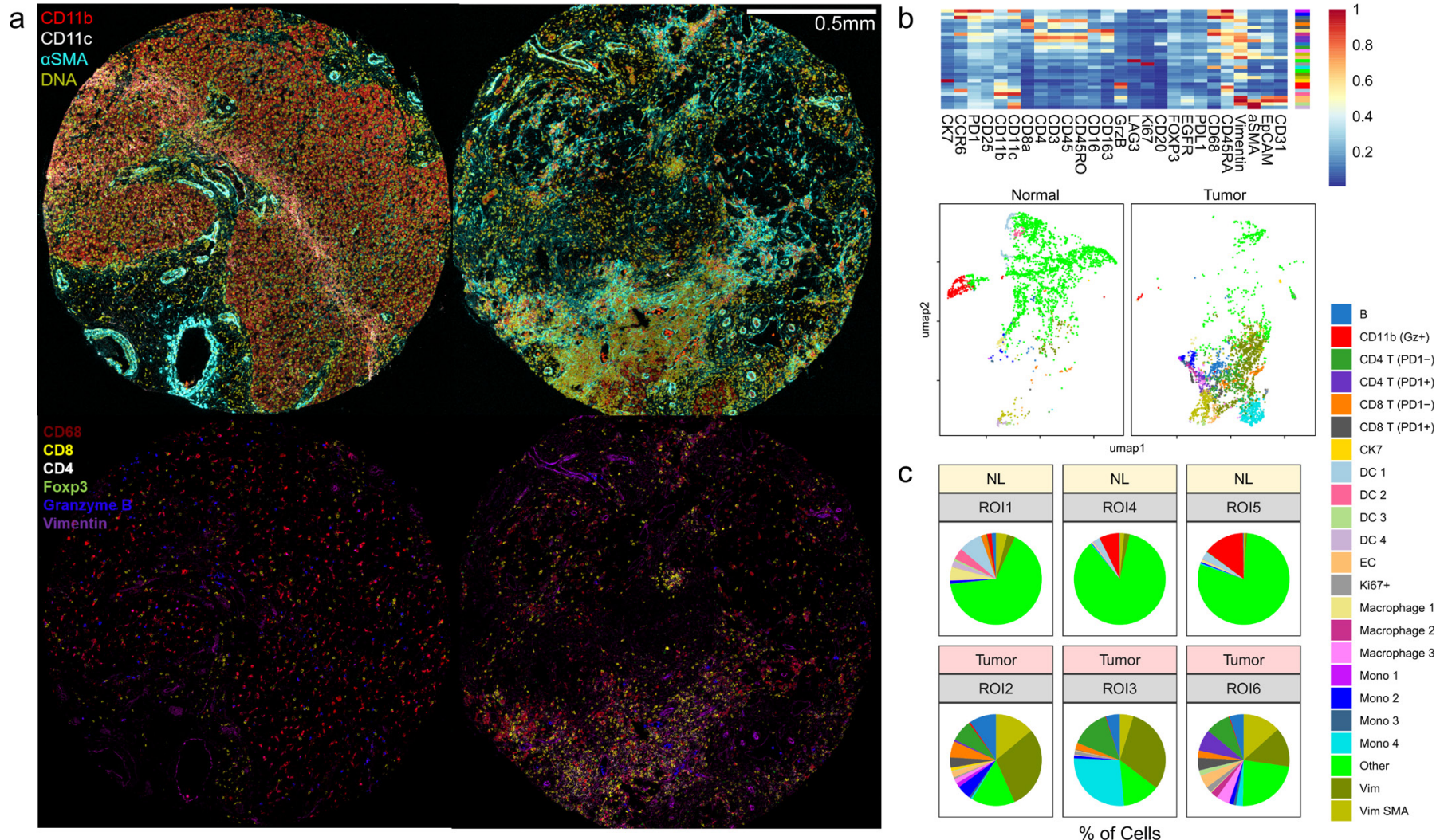

Figure 2 Neoadjuvant cabozantinib plus nivolumab results in dramatic immunological response in the tumor microenvironment. (A) Representative pictures of imaging mass cytometry of normal liver cores (left) and cores from surgically resected tumor samples post systemic therapy (right). Normal hepatocytes demonstrate mild CD11b positivity. Connective tissue deposition (aSMA) is higher within the tumor sample. Post-treatment core demonstrates a focus of dense immune infiltration (bottom right). (B) Results from FlowSOM clustering analysis is visualized as a heatmap and UMAP. (C) Tumor microenvironment profiles in three normal liver regions and three post-treatment tumor regions are compared. A total of 20,631 cells for normal and 39,066 cells for tumor regions are analyzed. aSMA, alpha-smooth muscle actin; DC, dendritic cells; EC, endothelial cells; Gz, granzyme B; Mono, monocytes; Vim, vimentin.

returned as clear margins with significant treatment effect. One of the lesions was $100 \%$ necrotic; the other lesion was more than $80 \%$ necrotic and residual areas of tumor were associated with a brisk immune infiltration (figure 1C). He did not receive any adjuvant therapy, but did receive surveillance imaging with CT scans every 3 months. He remains without disease recurrence (online supplemental figure 1) with excellent performance status (Eastern Cooperative Oncology Group (ECOG) Scale: 0 ), normal liver function, and a normal AFP over 2 years from presentation (online supplemental table 1).

\section{IMMUNOLOGIC ANALYSIS}

To understand the immunologic profiles underlying the clinical response, immune infiltration within the tumor microenvironment (TME) was first evaluated with multiplexed imaging mass cytometry (IMC) (figure 2A; zoomed-in image, online supplemental figure 3). To maximize the area of analysis, data was acquired from three large diameter $(1.5 \mathrm{~mm})$ cores from the surgical tumor sample. Since the pre-treatment biopsy was only a core biopsy sample, a normal liver sample was used as an independent reference. Clustering analysis with
FlowSOM algorithm of the single cells segmented from the images revealed markedly high abundances of immune cells, including CD4+T, CD8+T, and myeloid populations (figure 2B,C). We further validated the treatment-mediated increase in the presence of immune cells in the TME by comparing immune cell densities with single immunohistochemistry against CD3, CD8, and CD20 (online supplemental figure 3). As expected, the treatment led to a substantial increase in CD8 and CD20 positive cells. To delineate whether these cell types exhibited particular spatial relationships, two analytical methods were used on detected immune cell type patterns from the IMC data set (figure 3): neighborhood analysis, that is, how each cell type spatially relates to other cell types, performed using histoCAT; ${ }^{16}$ and autocorrelation statistics, that is, whether each cellular pattern is distributed in a coordinated manner, enabled by quantitative cell pattern detection with CoGAPS. Within the treated HCC, a significantly organized cellular neighborhood among CD8+T cell, CD4+T cell, B cell, and myeloid cell patterns were present. Also, spatial proximity between a stromal pattern (alpha-smooth muscle actin, aSMA) and the prominent immune cell neighborhood was notable. 

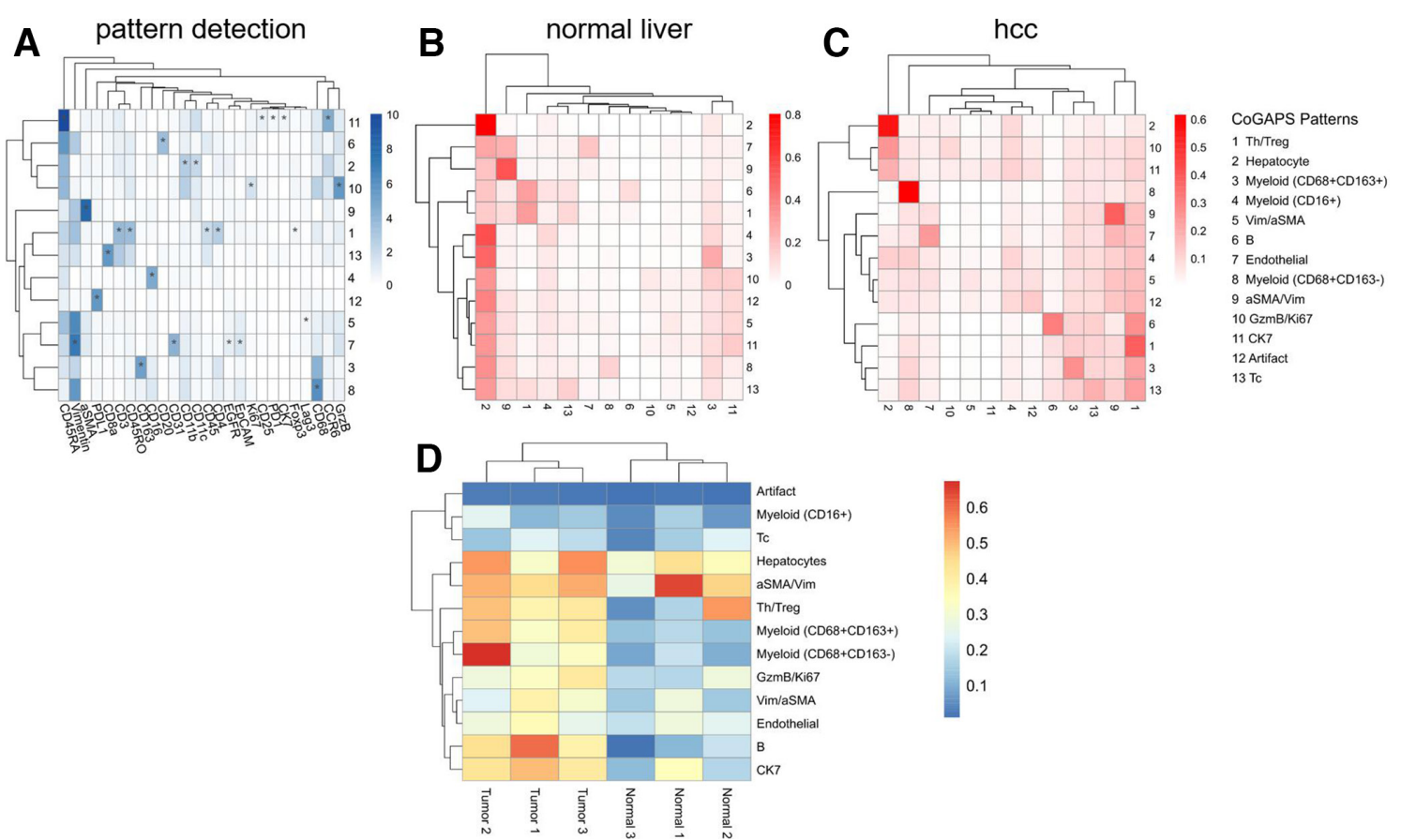

Figure 3 Neighborhood analysis of normal and post-treatment liver cores identifies distinct spatial neighborhoods in hepatocellular carcinoma treated with a combination of cabozantinib and nivolumab. (A) Pattern detection using Coordinated Gene Activity in Pattern Sets (CoGAPS) recapitulated major clusters identified by FlowSOM. Resulting pattern weights for each marker are shown as a heatmap. Markers that are driving the patterns according to the CoGAPS PatternMarker statistic are also marked with asterisks. (B and C) Heatmap of the cellular neighborhoods in normal liver (B) and HCC (C) regions. (D) Results of spatial autocorrelation for three normal liver and three HCC regions. GzmB, granzyme B; Tc, cytotoxic T cells; Th/Treg, helper $T$ cells/regulatory $T$ cells; Vim, vimentin.

Strong spatial autocorrelations were noted for immune cell patterns within the treated tumor microenvironment, especially for $\mathrm{B}$ cells, helper $\mathrm{T}$ cells, regulatory $\mathrm{T}$ cells, and CD68+CD163- myeloid cells. As expected, neighborhoods were only centered around hepatocytes and no significant autocorrelations could be identified in the normal liver. These results suggested that not only does cabozantinib-nivolumab combination lead to successful immune cell recruitment but also that the co-infiltration of lymphoid and myeloid cells occurs in a non-random, coordinated manner in relation to each other and to the stroma.

To evaluate the systemic immunologic effects related to combination of cabozantinib and nivolumab, we also assessed the peripheral immune profiles at baseline and post-treatment settings by suspension mass cytometry (CyTOF). Also, to compare the findings with the local immune response, we concurrently profiled the tumorinfiltrating immune cells using the same CyTOF panel (figure 4). In general, T cell abundances at earlier differentiation states, for example, naive and central memory, decreased while effector memory and effector populations increased. Furthermore, marked systemic promotion of effector cytotoxic $\mathrm{T}$ cells mirrored the dominant cell type present locally. There was also an overall decrease in regulatory T cells. Most notably, the relative abundance of natural killer (NK) cells increased with therapy.

All methods for the immunological analysis are described in detail in the online supplemental information).

\section{DISCUSSION}

The use of systemic therapies in HCC are typically restricted to patients with advanced HCC, or intermediate stage HCC that is not amenable to locoregional therapies. This first case provides support for the idea that patients who have HCC that is beyond standard resection criteria can be downstaged with upfront use of novel and highly active systemic therapy, with long-term disease-free survival. Additional research is needed to identify predictive biomarkers to select patients for the approach described in this case. The described case is consistent with the observations of Kaseb and colleagues, who demonstrated a high rate of pathologic responses in patients receiving neoadjuvant nivolumab with or without ipilimumab. ${ }^{17}$ However, in contrast to this prior report, our patient had unresectable disease at presentation, and demonstrates the potential for tumor downstaging in addition to pathologic responses. Furthermore, we are the first to test the combination of a TKI with immunotherapy in the preoperative setting in HCC. Based on this experience, the results of our phase $1 \mathrm{~b}$ are highly anticipated. It is worth noting, however, that this is a single case experience, and in fact, there have been extraordinary responses to even sorafenib, with which curative resection was performed successfully for locally advanced disease at presentation. $^{18}$

The use of systemic therapy in the neoadjuvant setting offers a tremendous research opportunity to better elucidate mechanisms of response and resistance in HCC. In 

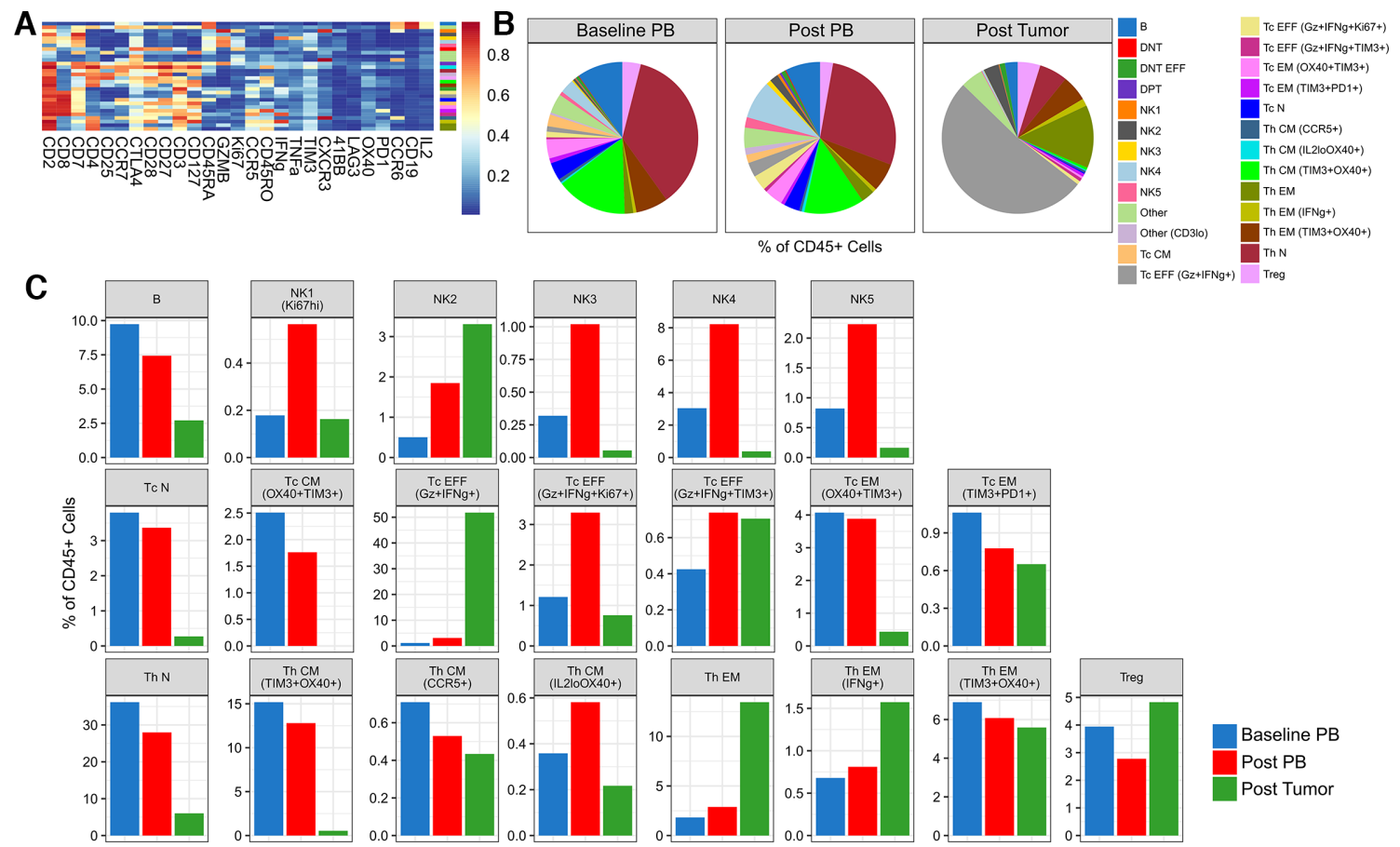

Figure 4 Differences in the immune profiles of baseline and post-treatment specimens are revealed by CyTOF. (A) Heatmap of the normalized expression for all markers used during FlowSOM clustering for all CD45+ cells acquired from baseline and posttreatment samples using a T cell-oriented CyTOF panel. The color bar to the right of the expression heatmap describes how each cell type has been annotated. (B) Pie and (C) bar plots showing the differences in abundance of the resulting immune cell clusters as a percentage of total CD45+ cells, comparing baseline peripheral blood (PB), post-treatment PB, and post-treatment surgical tumor sample from the patient. CM, central memory; DNT, double negative T cells; DPT, double positive T cells; EFF, effector; EM, effector memory; N, naïve; NK, natural killer; Tc, cytotoxic T cells; Th, helper T cells; Treg, regulatory T cells.

addition to characterizing an inflamed TME by robust presence of immune cells, the importance of spatial relationships among the infiltrating immune cells are increasingly being recognized. Recent studies including our own have reported the presence of distinctive immune cell aggregates, for example, tertiary lymphoid structures, within the TME in the context of immunotherapy. ${ }^{1920}$ Our analysis demonstrating high neighborhood and autocorrelation statistics among the lymphoid cells lend further support for the importance of such structures in immunologically active TME for therapeutic efficacy. Moreover, our multiplexed imaging with IMC added an additional depth to these observations that important spatial relationships also involve myeloid cells and stromal components. We also identified key systemic immunologic changes induced by the combination of cabozantinib and nivolumab. T cell related changes are consistent with the well-established effects of anti-PD1 therapies in promoting T cell activation. ${ }^{21}{ }^{22}$ It is, however, unclear as to why CD8+ cell density but not CD3+ cell density increases when comparing post-treatment versus pre-treatment settings. Since CD3-positivity includes a variety of $\mathrm{T}$ cell subtypes including regulatory $\mathrm{T}$ cells, one can speculate that the treatment is able to influence which functional subtype of $\mathrm{T}$ cells would dominate within the TME. Regarding NK cells, interestingly, there were notable increases in their abundance, supporting the hypothesis that this combination may elicit antitumor effects in part through the action of NK cells. ${ }^{23}$ Overall, further validation of these trends observed is anticipated pending the analysis of the entire trial. Methodologically, our study also demonstrates for the first time that CoGAPS algorithm enables the following two applications: (1) CoGAPS can recover cell type patterns from mass cytometry data sets that are consistent with FlowSOM and (2) CoGAPS pattern intensities for each cell provides continuous data that can be leveraged for autocorrelation statistics. The results from this first patient will be significantly enhanced by the final results of the trial and thus analysis of responders versus non-responders.

In conclusion, the striking clinical response to the novel combination of cabozantinib and nivolumab observed in this patient's scenario suggests that unresectable HCC may warrant consideration for attempted downstaging with effective preoperative systemic therapy. Our demonstrated approach to perform an unbiased integrated analysis of both local and systemic immune responses with complementary methodologies will be applied to every patient in the ongoing trial to accelerate discovery.

\section{Author affiliations}

${ }^{1}$ Department of Oncology, Johns Hopkins Medicine Sidney Kimmel Comprehensive Cancer Center, Baltimore, Maryland, USA

${ }^{2}$ Division of Biostatistics and Bioinformatics, Johns Hopkins Medicine Sidney Kimmel Comprehensive Cancer Center, Baltimore, Maryland, USA

${ }^{3}$ Department of Pathology, Johns Hopkins University School of Medicine, Baltimore, Maryland, USA 
${ }^{4}$ McKusick-Nathans Institute of Genetic Medicine, Johns Hopkins University School of Medicine, Baltimore, Maryland, USA

${ }^{5}$ Department of Radiology and Radiological Science, Johns Hopkins University School of Medicine, Baltimore, Maryland, USA

${ }^{6}$ Department of Surgery, Johns Hopkins University School of Medicine, Baltimore, Maryland, USA

\section{Twitter Won Jin Ho @wonjinho1}

Acknowledgements Mass cytometry data were acquired at the University of Maryland School of Medicine Center for Innovative Biomedical Resources (CIBR) Flow Cytometry and Mass Cytometry Core Facility, Baltimore, Maryland.

Contributors Conception and design: $\mathrm{M}$ Yarchoan. Development and methodology: W Ho, G Sharma, G Stein-0'Brien, EJ Fertig, G Mo, J Durham, EM Jaffee, and M Yarchoan. Acquisition of data: W Ho, G Mo, A Popovic, M Weiss, Q Zhu, R Anders, and M Yarchoan. Analysis and interpretation of data: W Ho, A Popovic, I Kamel, G Sharma, EJ Fertig, Q Zhu, R Anders, EM Jaffee, and M Yarchoan. Writing, review, and/or revision of the manuscript: All authors. Administrative, technical, or material support: J Durham, EM Jaffee, and M Yarchoan.

Funding Funding for this clinical trial were provided by Exelixis and Bristol Myers Squibb (to MY). Additional research support was provided by the National Cancer Institute Specialized Program of Research Excellence (SPORE) in Gastrointestinal Cancers (P50 CA062924), the NIH Center Core Grant (P30 CA006973), and the Emerson Collective Cancer Research Fund (640183).

Competing interests WH is a co-inventor of patents with potential for receiving royalties from Rodeo Therapeutics unrelated to the current study. MY reports receiving a commercial research grant from Bristol Myers Squibb, Exelixis, and Merck \& $\mathrm{Co}$ and is a consultant/advisory board member for Eisai and Exelixis. EJF is a consultant for Champions Oncology. EMJ reports receiving a commercial research grant from Bristol Myers Squibb, Aduro Biotech, and Amgen, has ownership interest (including stock, patents, and so on) in Aduro Biotech, and is a consultant/advisory board member for CStone, Dragonfly, Genocea, and Adaptive Biotechnologies.

\section{Patient consent for publication Not required.}

Ethics approval The evaluation of clinical samples was performed in accordance with the protocols approved by the Johns Hopkins Institutional Review Board (IRB). All specimens were obtained with written patient consent (IRB00149350).

Provenance and peer review Not commissioned; externally peer reviewed.

Supplemental material This content has been supplied by the author(s). It has not been vetted by BMJ Publishing Group Limited (BMJ) and may not have been peer-reviewed. Any opinions or recommendations discussed are solely those of the author(s) and are not endorsed by BMJ. BMJ disclaims all liability and responsibility arising from any reliance placed on the content. Where the content includes any translated material, BMJ does not warrant the accuracy and reliability of the translations (including but not limited to local regulations, clinical guidelines, terminology, drug names and drug dosages), and is not responsible for any error and/or omissions arising from translation and adaptation or otherwise.

Open access This is an open access article distributed in accordance with the Creative Commons Attribution Non Commercial (CC BY-NC 4.0) license, which permits others to distribute, remix, adapt, build upon this work non-commercially, and license their derivative works on different terms, provided the original work is properly cited, appropriate credit is given, any changes made indicated, and the use is non-commercial. See http://creativecommons.org/licenses/by-nc/4.0/.

\section{ORCID iD}

Won Jin Ho http://orcid.org/0000-0003-2644-5086

\section{REFERENCES}

1 Villanueva A. Hepatocellular carcinoma. N Engl J Med 2019;380:1450-62.

2 Sasaki A, Iwashita Y, Shibata K, et al. Preoperative transcatheter arterial chemoembolization reduces long-term survival rate after hepatic resection for resectable hepatocellular carcinoma. Eur J Surg Oncol 2006;32:773-9.

3 Wu CC, Ho YZ, Ho WL, et al. Preoperative transcatheter arterial chemoembolization for resectable large hepatocellular carcinoma: a reappraisal. Br J Surg 1995;82:122-6.

4 Amisaki M, Honjo S, Morimoto M, et al. The negative effect of preoperative transcatheter arterial chemoembolization on long-term outcomes for resectable hepatocellular carcinoma: a propensity score matching analysis. Yonago Acta Med 2016;59:270-278.

5 Choi SB, Kim KS, Park YN, et al. The efficacy of hepatic resection after neoadjuvant transarterial chemoembolization (TACE) and radiation therapy in hepatocellular carcinoma greater than $5 \mathrm{~cm}$ in size. J Korean Med Sci 2009;24:242-7.

6 Uchida M, Kohno H, Kubota $\mathrm{H}$, et al. Role of preoperative transcatheter arterial oily chemoembolization for resectable hepatocellular carcinoma. World J Surg 1996;20:326-31.

7 Cheng A-L, Qin S, Ikeda M, et al. IMbrave150: efficacy and safety results from a $\mathrm{pH}$ III study evaluating atezolizumab (atezo) + bevacizumab (bev) vs sorafenib (SOR) as first treatment (tx) for patients (PTS) with unresectable hepatocellular carcinoma (HCC). Annals of Oncology 2019;30:ix186-7.

8 Llovet J, Shepard KV, Finn RS, et al. A phase lb trial of lenvatinib (LEN) plus pembrolizumab (PEMBRO) in unresectable hepatocellular carcinoma (uHCC): updated results. Annals of Oncology 2019;30:v286-7

9 Finn RS, Qin S, Ikeda M, et al. Atezolizumab plus bevacizumab in unresectable hepatocellular carcinoma. $N$ Engl J Med 2020;382:1894-905.

10 Zhang J, Huang M, Cai Y, et al. Neoadjuvant chemotherapy with $\mathrm{mFOLFOXIRI}$ without routine use of radiotherapy for locally advanced rectal cancer. Clin Colorectal Cancer 2019;18:238-44.

11 Peyton CC, Tang D, Reich RR, et al. Downstaging and survival outcomes associated with neoadjuvant chemotherapy regimens among patients treated with cystectomy for muscle-invasive bladder cancer. JAMA Oncology 2018;4:1535-42.

12 Jackson HW, Fischer JR, Zanotelli VRT, et al. The single-cell pathology landscape of breast cancer. Nature 2020;578:615-20.

13 Stein-O'Brien GL, Clark BS, Sherman T, et al. Decomposing cell identity for transfer learning across cellular measurements, platforms, tissues, and species. Cell Syst 2019;8:395-411.

14 Van Gassen S, Callebaut B, Van Helden MJ, et al. FlowSOM: using self-organizing maps for visualization and interpretation of cytometry data. Cytometry A 2015;87:636-45.

15 Llovet JM, Fuster J, Bruix J, et al. The Barcelona approach: diagnosis, staging, and treatment of hepatocellular carcinoma. Liver Transpl 2004;10:S115-20.

16 Schapiro D, Jackson HW, Raghuraman S, et al. histoCAT: analysis of cell phenotypes and interactions in multiplex image cytometry data. Nat Methods 2017;14:873-6.

17 Kaseb AO, Carmagnani Pestana R, Vence LM, et al. Randomized, open-label, perioperative phase II study evaluating nivolumab alone versus nivolumab plus ipilimumab in patients with resectable HCC. Journal of Clinical Oncology 2019;37:185.

18 Irtan S, Chopin-Laly X, Ronot M, et al. Complete regression of locally advanced hepatocellular carcinoma induced by sorafenib allowing curative resection. Liver Int 2011;31:740-3.

19 Stein JE, Lipson EJ, Cottrell TR, et al. Pan-Tumor Pathologic Scoring of Response to PD-(L)1 Blockade 2019.

20 Helmink BA, Reddy SM, Gao J, et al. B cells and tertiary lymphoid structures promote immunotherapy response. Nature 2020;577:549-55.

21 Freeman GJ, Long AJ, Iwai Y, et al. Engagement of the PD-1 immunoinhibitory receptor by a novel B7 family member leads to negative regulation of lymphocyte activation. J Exp Med 2000;192:1027-34.

22 Blank C, Brown I, Peterson AC, et al. PD-L1/B7H-1 inhibits the effector phase of tumor rejection by $T$ cell receptor (TCR) transgenic CD8+ T cells. Cancer Res 2004;64:1140-5.

23 Verzoni E, Ferro S, Procopio G, et al. Potent natural killer (NK) and myeloid blood cell remodeling by cabozantinib (Cabo) in pre-treated metastatic renal cell carcinoma (mRCC) patients (PTS). Annals of Oncology. ; 2018;29:viii312. 